\title{
Widespread Sensitivity to Looming Stimuli and Small Moving Objects in the Central Complex of an Insect Brain
}

\author{
Ronny Rosner and Uwe Homberg \\ Philipps-Universität Marburg, Tierphysiologie, D-35032 Marburg, Germany
}

In many situations animals are confronted with approaching objects. Depending on whether the approach represents a potential threat or is intended during a goal-oriented approach, the adequate behavioral strategies differ. In all of these cases the visual system experiences an expanding or looming shape. The neuronal machinery mediating looming elicited behavioral responses has been studied most comprehensively in insects but is still far from being fully understood. It is particularly unknown how insects adjust their behavior to objects approaching from different directions. A brain structure that is thought to play an important role in spatial orientation in insects is the central complex (CC). We investigated whether CC neurons process information about approaching objects on a collision course. We recorded intracellularly from CC neurons in the locust Schistocerca gregaria during visual stimulation via lateral LCD screens. Many neurons in the locust CC, including columnar and tangential neurons, were sensitive to looming stimuli. Some of the neurons also responded to small moving targets. Several cell types showed binocular responses to looming objects, and some neurons were excited or inhibited depending on which eye was stimulated. These neurons may, therefore, detect the gross azimuthal direction of approaching objects and may mediate directional components of escape or steering movements.

\section{Introduction}

For spatial orientation animals depend on information about the structure of the surrounding world. Spatial information can be provided by various sensory systems, but in many insects the visual system plays a major role. Vision enables insects to choose and maintain a particular direction when flying or walking, to effectively avoid approaching predators or obstacles, to fixate and pursue mobile targets, and to discriminate shapes or complex patterns (Wehner, 1981; Giurfa and Menzel, 1997; Srinivasan et al., 1999; Egelhaaf, 2006). The perception of expanding images, experienced during object approach, contributes to depth and distance perception (Kirchner and Lengler, 1994; Wicklein and Sejnowski, 2001) and may trigger landing (Borst and Bahde, 1988) or escape responses. In locusts, the lobula giant movement detector/descending contralateral movement detector (LGMD/ DCMD) neurons mediate flight steering maneuvers and escape jumps in response to a looming threat (Simmons et al., 2010; Fotowat and Gabbiani, 2011).

Responses to looming stimuli are highly directional, in case of escape and hiding responses away from the stimulus (Hassenstein and Hustert, 1999; Santer et al., 2005; Card and Dickinson,

\footnotetext{
Received Nov. 20, 2012; revised March 22, 2013; accepted March 24, 2013.

Author contributions: R.R. and U.H. designed research; R.R. performed research; R.R. and U.H. contributed unpublished reagents/analytic tools; R.R. analyzed data; R.R. and U.H. wrote the paper.

This work was supported by Deutsche Forschungsgemeinschaft grants HO 950/16-3 and HO 950/21-1. We are grateful to Ronny Heidasch and Joss von Hadeln for providing reconstructions of the TUx, TUy, TUz, TL3, CU4, and CPU1 neurons; Dr. Erich Buchner for providing the synapsin antibody; and Martina Kern und Jutta Seyfarth for raising desert locusts.

The authors declare no competing financial interests.

Correspondence should be addressed to Ronny Rosner, Fachbereich Biologie, Tierphysiologie, Philipps-Universität Marburg, Karl-von-Frisch-Straße 8, D-35032 Marburg, Germany. E-mail: ronny.rosner@biologie.uni-marburg.de.

DOI:10.1523/JNEUROSC1.5390-12.2013

Copyright $\odot 2013$ the authors $\quad 0270-6474 / 13 / 338122-12 \$ 15.00 / 0$
}

2008). The neuronal substrate that allows insects to steer their responses into a particular direction, however, is unknown. A candidate brain structure for mediating directional movements is the central complex (CC). The CC comprises a group of neuropils spanning the brain midline and consists of the protocerebral bridge $(\mathrm{PB})$, the upper and lower divisions of the central body (CBU, CBL), and the paired noduli (Homberg, 1987; Heinze and Homberg, 2008). In many insects studied, the CC is dominated by visual input (Homberg, 2008): in locusts it holds an internal representation of sky compass directions (Heinze and Homberg, 2007), and in flies and cockroaches it plays a key role in spatial orientation during walking and climbing (Neuser et al., 2008; Triphan et al., 2010; Ritzmann et al., 2012).

The blue sky shows a prominent pattern of polarized light, which is used by many insects for spatial orientation (Horváth and Varjú, 2004). In locusts and monarch butterflies, many neurons ramifying in the $\mathrm{PB}$, the $\mathrm{CBL}$, and the noduli of the $\mathrm{CC}$ are sensitive to the plane of oscillation of dorsally presented polarized light (Heinze and Reppert, 2011; Homberg et al., 2011). In contrast, polarization sensitivity is conspicuously absent in neurons of the CBU. Instead, CBU neurons were speculated to modify the response properties of polarization-sensitive neurons depending on the behavioral state of the insect (Homberg, 1994; Heinze and Homberg, 2009). Additionally, CBU neurons in flies (Liu et al., 2006; Phillips-Portillo, 2012) and locusts (Homberg, 1994) process unpolarized visual information.

We investigated whether neurons of the locust CC are sensitive to looming stimuli and small translating objects. We found that various cell types responded vigorously to expanding shapes and in some cases to translating objects. Many neurons ramified in the CBU, but cell types in other parts of the CC were also looming sensitive. Specific response properties of some of the 


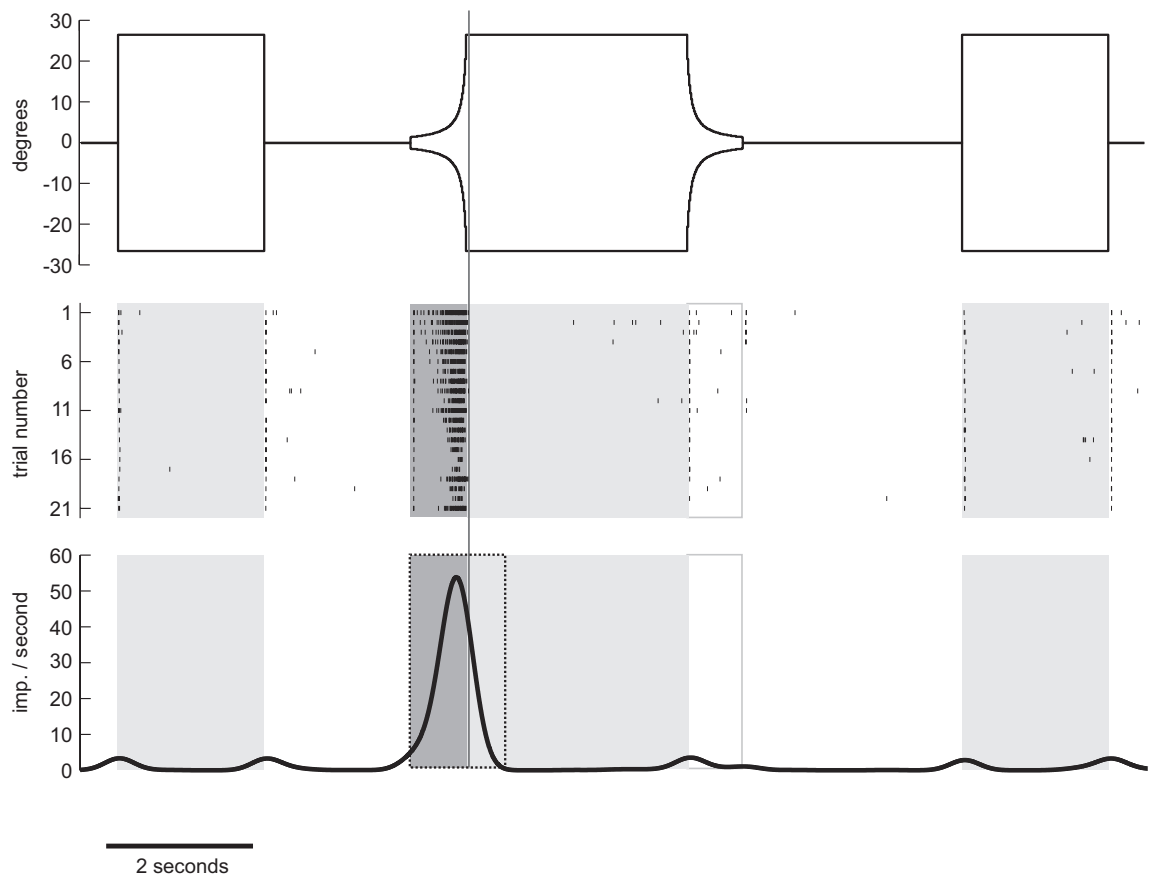

Figure 1. Stimulus regime and evaluation of looming sensitivity illustrated for a recording from the DCMD neuron. Top, Stimulus sequence of a single trial. The vertical extent of a black disc displayed in the middle of a bright 8 inch LCD-screen is shown as a function of time. Positive (negative) values indicate angular extent upward (downward) from screen center as seen from the locust. The trial starts with a $500 \mathrm{~ms}$ blank screen interval, then a disc with a diameter of $53^{\circ}$ is displayed for $2 \mathrm{~s}$. After another blank screen interval of $2 \mathrm{~s}$ an approaching object on a collision course is simulated by means of a looming disc. The simulated object (diameter $10 \mathrm{~cm}$ ) starts at a distance of $2 \mathrm{~m}$ and approaches the locust with a velocity of $2.5 \mathrm{~m} / \mathrm{s}$, yielding a time to collision of the object of $800 \mathrm{~ms}$ after simulation onset. The simulated approach ends abruptly after $760 \mathrm{~ms}$ at a distance of $10 \mathrm{~cm}\left(53^{\circ}\right.$ angular extent as seen from the locust). The simulated object stays stationary for 3 s and then draws back with the same speed and vanishes at the same distance as it had started to approach. Subsequently the screen stays blank for $3 \mathrm{~s}$. Finally, the disc is again displayed for $2 \mathrm{~s}$ and afterward the screen is blank for another $500 \mathrm{~ms}$. The middle panel shows the occurrence of action potentials generated by a DCMD neuron in response to 21 repetitions of the stimulus protocol. The first seven repetitions were shown in immediate succession (intertrial distance $10-12 \mathrm{~s}$ ). The remaining trials were shown with no strict time regime and were interrupted by the presentation of other stimuli. Bottom, Mean of Gaussian-filtered ( $S D=200 \mathrm{~ms}$ ) spike trains. Boxes in the middle and bottom represent time periods with presentation of stationary (shaded light gray), expanding (shaded dark gray), and contracting (box framed in gray) disc. Gray vertical line across all sections illustrates time of collision and dotted frame in bottom illustrates evaluation window for looming sensitivity (see text). The DCMD neuron is strongly excited during presentation of the expanding disc.

neurons make them promising candidates for mediating directional behaviors away from or toward approaching objects.

\section{Materials and Methods}

Preparation. Experiments were performed on adult desert locusts (Schistocerca gregaria) of either sex. Animals were reared under crowded conditions at a constant temperature of $28^{\circ} \mathrm{C}$ and a $12 \mathrm{~h}$ light/dark cycle. Animals were mounted on custom-made holders and restrained in movements by removing or shortening wings and legs and immobilizing the remaining stumps, the head, and mouthparts by wax. A hole was cut into the anterior head capsule to allow access to the central brain. The ocellar nerves were cut to ensure that visual stimuli evoke neuronal responses only via the compound eyes. Fat surrounding the brain and tracheal sacs was removed. The neural sheath was stripped away at the region where the recording electrode was inserted. The gut was removed to prevent pumping movements, and to further stabilize the brain a wire platform supported the brain from posterior. During recording of neural activity the brain was submerged in locust saline (Clements and May, 1974).

Electrophysiology. We recorded intracellularly from 38 centralcomplex neurons using borosilicate micropipettes drawn on a microelectrode puller (Sutter Instruments P-97). The tips of the electrodes were filled with $4 \%$ Neurobiotin (Vector Laboratories) in $1 \mathrm{~m} \mathrm{KCl}$ and their shanks with $1 \mathrm{M} \mathrm{KCl}$. The electrodes had tip resistances of 100-180 M $\Omega$.
Signals were amplified (SEC5-LX amplifier; NPI), digitized (CED1401 micro; Cambridge Electronic Design), and stored using a PC with Spike2 software (Cambridge Electronic Design). Depolarizing current was applied to iontophoretically inject Neurobiotin immediately after recording and in some recordings in between the stimulus sequences.

Histology. After completing physiological recording, brains were dissected out of the head capsule and fixed overnight in a mixture of $4 \%$ paraformaldehyde, $0.25 \%$ glutaraldehyde, and $2 \%$ saturated picric acid in $0.1 \mathrm{M}$ phosphate buffer. The labeled neurons were made visible for confocal laser scanning microscopy (Leica TCS-SP5; Leica Microsystems) by treatment of the brains with $\mathrm{Cy} 3$-conjugated streptavidin (Dianova). The detailed procedure is described in Heinze and Homberg (2008). The anatomy of novel neurons was analyzed in detail. For this, brains were sectioned as described by Heinze and Homberg (2008). Briefly, brains were embedded in gelatin/albumin and then sectioned in slices of $130 \mu \mathrm{m}$ with a vibratingblade microtome (Leica VT1200 S, Leica Microsystems). The slices were treated with a monoclonal mouse antibody against synapsin I (SYNORF1; Klagges et al. 1996) provided by E. Buchner, Würzburg, to unveil brain neuropils. After incubation with the secondary antibody, goat anti-mouse conjugated to $\mathrm{Cy} 5$ (Cy5GAM; Jackson ImmunoResearch) and with Cy3-streptavidin the sections were dehydrated, cleared, and embedded in Permount. Finally brain sections were scanned with the confocal laser scanning microscope and the obtained image stacks were processed on a personal computer using Amira 5.3.3 software (Visage Imaging; see below).

Visual stimulation. Visual stimuli were presented via two 8 inch flat screen LCD monitors (Faytech) with a resolution of $800 \times 600$ pixels and driven at 60 or $75 \mathrm{~Hz}$. The monitors were located $7 \mathrm{~cm}$ laterally to the locust's head. Stimuli were written with MATLAB (MathWorks) using the Psychophysics Toolbox (Brainard, 1997; Pelli, 1997). Light from the displays was completely depolarized by diffuser sheets mounted in front of the displays.

We aimed to investigate whether CC neurons are sensitive to approaching objects that were simulated on the monitors by expanding ("looming") stimuli. We tested all neurons for their sensitivity to expanding discs or squares. To distinguish whether a neuron responded to the looming property or solely to a change in brightness or the presence of the object we presented nonlooming discs or squares in the same trials. In addition, we simulated the withdrawal of an object by a contracting disc or square. The stimulation scheme is illustrated in Figure 1. The angular extent $\alpha$ of the expanding shapes was calculated as a function of time $t$ during approach:

$$
\alpha(t)=2 \cdot \tan ^{-1} \frac{l}{v t}
$$

where $l$ and $v$ denote the object's half size and approaching velocity, respectively. We tested the effectiveness of this stimulation by extracellular recording from a well known looming-sensitive descending neuron without ramifications in or known connections to the CC, the DCMD neuron (Fig. 1).

We mainly tested black objects against a bright background, after preliminary experiments had shown that responses were much weaker or 
completely absent when using bright objects against a dark background in many CC neurons. The Michelson contrast of the objects was 0.97 calculated with luminances of $70 \mathrm{~cd} / \mathrm{m}^{2}$ (bright monitor) and $1 \mathrm{~cd} / \mathrm{m}^{2}$ (dark monitor) measured with an OptiCAL photometer (Cambridge Research Systems). The discs were always presented in the center of one of the two screens, whereas the squares were also shown approaching the animal from an angle deviating by $30^{\circ}$ from the screen center. In those cases, however, the squares were adjusted to be experienced by the locusts as being invariant in shape and approaching on a direct collision course. In the current account we pooled data acquired during the presentation of squares at different positions of the monitor. When squares and discs were applied during a recording we only evaluated the traces generated by the condition, which was applied more often resulting in the higher number of trials.

The simulated speed of approach was not identical in all experiments but ranged from 2 to $5 \mathrm{~m} / \mathrm{s}$. The half size to speed ratio was in the range of $10-25 \mathrm{~ms}$. Each cell was only tested with a single speed. The discs and squares stopped before the time of theoretical collision with the animals. The virtual distance at which the simulated object stopped varied from 42 to $200 \mathrm{~mm}$ between different recordings. However, an evaluation of response differences for looming stimuli simulating different velocities or approach distances is beyond the scope of the present study. In those cases in which the simulated approaching distance was $42 \mathrm{~mm}$ the discs were truncated in the last visible frame by the edges of the monitor.

Some neurons were also tested for their sensitivity to moving and stationary small squares and bars or stationary and drifting gratings. In some experiments we also applied polarized light stimuli, in addition to the stimuli presented via the two monitors, mainly to classify the recorded cells. In the current account we restrict our analysis to those unpolarized light stimuli that elicited strong responses in the locust CC.

Data analysis. Image data evaluation and visualization of the stained neurons was done with Amira software and Adobe Photoshop (Adobe Systems). A selection of neurons was three-dimensionally reconstructed as described previously (el Jundi et al., 2009). 3D reconstructions of the neurons were performed using the SkeletonTree tool (Schmitt et al., 2004).

In several preparations two or more neurons rather than a single neuron were stained. The likely reason for this is leakage of Neurobiotin from the electrode tip without applying depolarizing current to a cell. In those cases we assumed that the most intensely stained neuron was the one that we recorded from and stained actively. In some cases, physiological characteristics, especially sensitivity to polarized light, which is only found in certain cell types, provided additional evidence for the identity of the recorded neuron. The terms ipsilateral and contralateral are used with respect to the location of the soma. Putative input (output) arborizations were identified by their smooth (varicose) appearance (Heinze and Homberg, 2008).

Analysis of physiological data was done off-line in MATLAB. We extracted spike times from recording traces and created a vector of ones and zeros (bin size $1 \mathrm{~ms}$ ) for each trace representing the presence or absence of an action potential, respectively. Then we convolved the vectors with a Gaussian window to obtain an estimate of the spiking frequency. The filter window was $2000 \mathrm{~ms}$ in length. We used a relatively large SD of 200 ms because this smoothes out high background variabilities found in some neurons while facilitating the identification of stimulus-induced responses. The filtered traces were adjusted to have no phase shift in time as compared with the original spike trains.

The evaluation of looming experiments was done in the following way. First we categorized whether a cell was responsive to looming stimuli. For this we evaluated the spiking frequency during the course of an entire trial starting with a blank screen interval of $500 \mathrm{~ms}$ followed by the display, the looming, and the retraction of a disc (or square) in the succession as described in Figure 1 and ending with a 500 ms blank screen interval. A cell was regarded as looming sensitive if the spiking frequency peak or nadir occurred in a time interval ranging from the start of the looming stimulus until $500 \mathrm{~ms}$ after the prospective object collision time (Fig. 1). We applied this procedure for the mean response of a neuron and separately for the first trials of stimulation of the left eye and the right eye. We calculated the first trial responses in addition to the analysis of
Table 1. Sensitivity to looming stimuli and small moving objects in CC neurons

\begin{tabular}{|c|c|c|c|c|c|c|}
\hline Cell type & $\begin{array}{l}\text { Ipsilateral } \\
\text { first trial }\end{array}$ & $\begin{array}{l}\text { Contralateral } \\
\text { first trial }\end{array}$ & $\begin{array}{l}\text { Ipsilateral } \\
\text { mean }\end{array}$ & $\begin{array}{l}\text { Contralateral } \\
\text { mean }\end{array}$ & $\begin{array}{l}\text { Looming } \\
\text { sensitivity }\end{array}$ & $\begin{array}{l}\text { Small } \\
\text { object } \\
\text { sensitivity }\end{array}$ \\
\hline TUx, ${ }^{1}$ Fig. 2 & + & + & $+(4)$ & $+(3)$ & Yes & n.t. \\
\hline TUy, Fig. 3 & + & + & $+(3)$ & $+(5)$ & Yes & n.t. \\
\hline TUz, Fig. 4 & - & + & $-(5)$ & $+(4)$ & Yes & n.t. \\
\hline TU2 & 0 & + & $+(3)$ & $+(3)$ & Yes & n.t. \\
\hline GFS & 0 & 0 & $-(8)$ & $0(5)$ & Yes & n.t. \\
\hline GFS & 0 & 0 & $-(5)$ & $+(4)$ & Yes & Yes \\
\hline GFSL1 & 0 & + & $+(5)$ & $+(10)$ & Yes & n.t. \\
\hline GFSL2 & + & + & $+(3)$ & $+(3)$ & Yes & n.t. \\
\hline GFS/PoU1 & + & + & $+(4)$ & $+(2)$ & Yes & Yes \\
\hline GFS/PoU2 & 0 & - & $0(4)$ & $-(3)$ & Yes & n.t. \\
\hline PoU1, Fig. 5C-E & + & - & $+(3)$ & $-(3)$ & Yes & Yes \\
\hline PoU1, Fig. $5 A, B$ & + & 0 & $+(18)$ & $0(5)$ & Yes & No \\
\hline CU4, Fig. $6 A, B$ & - & + & $-(4)$ & $+(4)$ & Yes & n.t. \\
\hline CU4, ${ }^{2}$ Figs. $6 C, D, 9 B$ & + & + & $+(4)$ & $+(1)$ & Yes & Yes \\
\hline TL3 & 0 & - & $0(4)$ & $-(6)$ & Yes & n.t. \\
\hline CL1 & + & 0 & $+(7)$ & $0(7)$ & Yes & n.t. \\
\hline CL1 & + & n.t. & $+(3)$ & n.t. & Yes & No \\
\hline CL1 & + & + & $0(8)$ & $0(5)$ & Yes & Yes \\
\hline CL1 & 0 & 0 & $0(2)$ & $0(3)$ & No & n.t. \\
\hline CL1 & 0 & + & $0(5)$ & $+(2)$ & Yes & Yes \\
\hline CL1 & + & + & $0(3)$ & $+(2)$ & Yes & No \\
\hline CL1 & + & + & $+(6)$ & $+(7)$ & Yes & No \\
\hline CL1 & 0 & + & $+(3)$ & $+(4)$ & Yes & n.t. \\
\hline CL1 & + & + & $+(6)$ & $+(13)$ & Yes & No \\
\hline $\mathrm{CL} 1$ & + & + & $+(7)$ & $+(6)$ & Yes & n.t. \\
\hline CL1 & + & 0 & $+(5)$ & $0(3)$ & Yes & n.t. \\
\hline $\mathrm{CL}^{3}$ & + & 0 & $+(5)$ & $0(4)$ & Yes & No \\
\hline CL1 & 0 & 0 & $-(3)$ & $0(3)$ & Yes & Yes \\
\hline $\mathrm{CL} 1$ & - & + & $-(3)$ & $0(2)$ & Yes & n.t. \\
\hline CL1 & - & - & $-(6)$ & $-(5)$ & Yes & n.t. \\
\hline TB2 & + & + & $+(2)$ & $+(2)$ & Yes & Yes \\
\hline TB2 & + & 0 & $+(3)$ & $0(2)$ & Yes & Yes \\
\hline TB1, Fig. 9A & + & + & $+(8)$ & $+(6)$ & Yes & Yes \\
\hline CPU1 & 0 & - & $0(3)$ & $-(2)$ & Yes & n.t. \\
\hline CPU1 & - & n.t. & $-(4)$ & n.t. & Yes & n.t. \\
\hline CPU1 & - & - & $-(4)$ & $-(4)$ & Yes & n.t. \\
\hline CPU1, Fig.7 & - & - & $-(3)$ & $-(3)$ & Yes & Yes \\
\hline PoU1/CPU1 & n.t. & - & n.t. & $-(3)$ & Yes & No \\
\hline
\end{tabular}

Neurons were classified as looming sensitive if the peak $(+)$ or nadir $(-)$ of spiking activity occurred within a time window ranging from the start of the looming stimulus until $500 \mathrm{~ms}$ after the prospective object collision time, either in the first trial or in the mean response of all trials on either eye. Trial number is given in brackets. 0, peak or nadir activity occurred outside the critical time window. Neurons were classified as sensitive to small translating objects if the peak or nadir spiking rate occurred in response to the first translation in a sequence of object translations and rests. n.t., not tested. Nomenclature of cell types from Homberg (1994), Müller et al. (1997), Homberg et al. (1999), and Heinze and Homberg (2009). In two cases it was unclear whether we recorded from a GFS or a colabeled intrinsic neuron of the CBU (PoU1 neuron, respectively, PoU2 neuron; Heinze and Homberg, 2008). In one case it was unclear whether we recorded from a CPU1 or a colabeled PoU1 neuron. The input ramifications of the CPU1 neuron were contralateral to the input of PoU1. We classified regarding to the CPU1 neuron. ${ }^{1}$ The soma got lost during tissue processing, classified regarding to location of input (smooth) ramifications. ${ }^{2}$ The CU4 neuron also shown in Fig. 6C (responses in Figs. 6D and $9 B$ ) is marked as sensitive to the small objects although it does not fulfill the criterion (see text). ${ }^{3}$ Ipsilateral and contralateral could not be determined because several neurons of the same type were stained: arbitrary classification.

the mean response because some cells showed strong adaptation effects covering looming sensitivity in the mean.

\section{Results}

The data are based on 38 intracellular recordings combined with Neurobiotin tracer injection from neurons with ramifications in the CC of the locust brain. Visual stimuli were applied via two LCD monitors positioned laterally from the left and right eye. Responses to looming stimuli and small translating objects were found in a wide variety of neuronal cell types with ramifications in the $\mathrm{CBU}$ and $\mathrm{CBL}$ and in the $\mathrm{PB}$. In some of the recordings we also tested moving or stationary bars or gratings, but responses of 

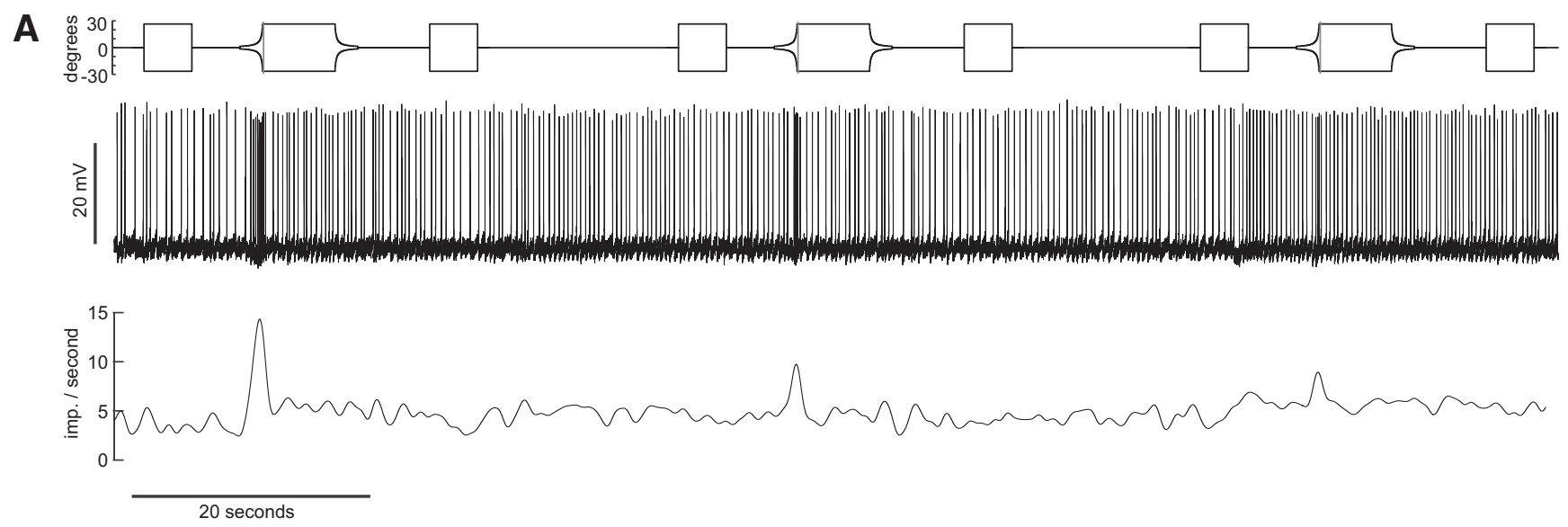

B

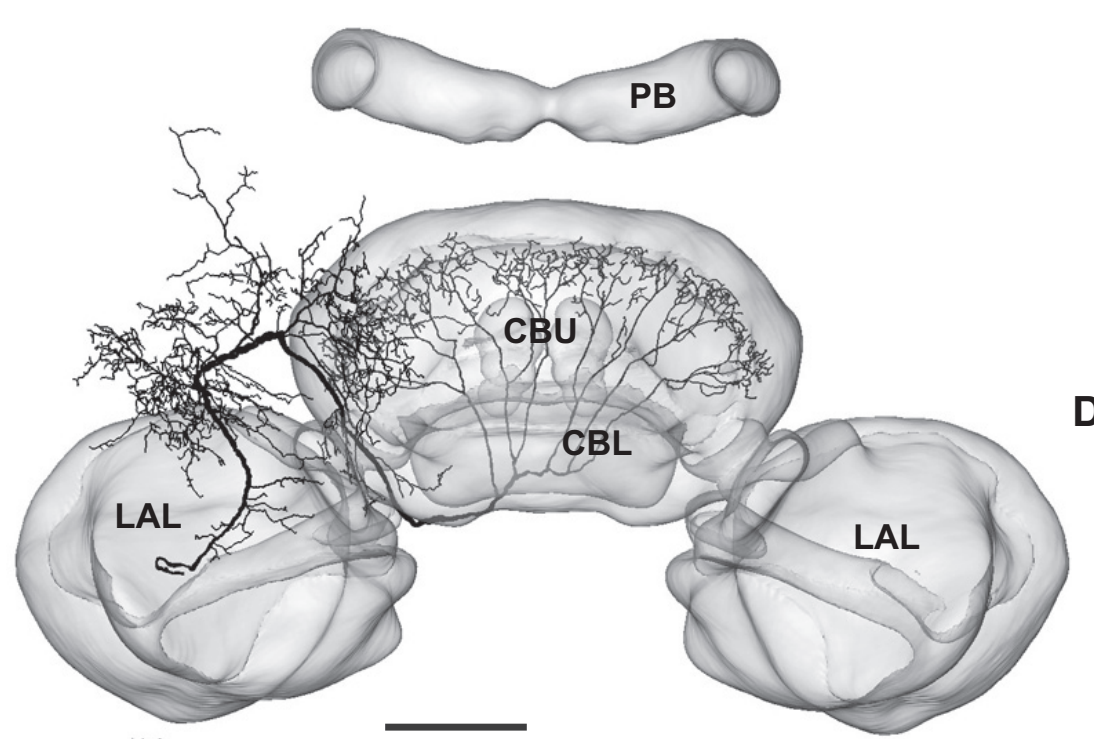

C

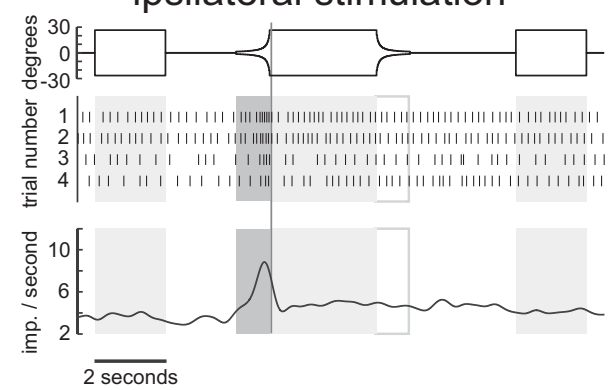

D

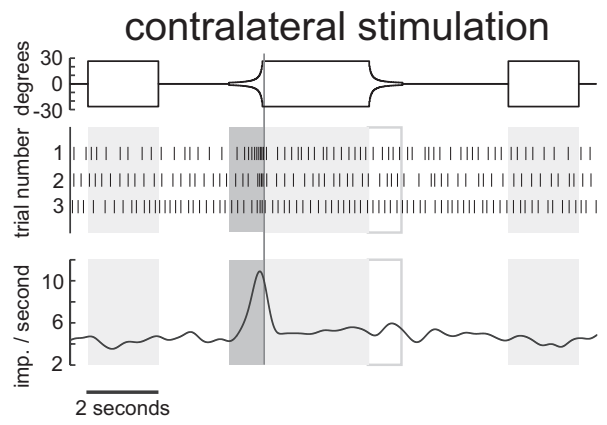

Figure 2. Looming-sensitive TUx tangential neuron of the CBU. A, Original recording trace of TUx neuron (middle trace, slow fluctuations removed by subtracting running average) during stimulation of the contralateral eye. Lower trace shows Gaussian-filtered spike train. Upper trace shows angular extent of disc as seen by the locust as a function of time. $\boldsymbol{B}$, Frontal reconstruction of the TUx neuron registered into the standard central complex of the locust brain (el Jundi et al., 2010). The neuron has wide, presumably input ramifications in the superior medial protocerebrum and fan-shaped axonal ramifications in layer II of the CBU. The soma of the neuron got lost during tissue processing. Scale bar, $100 \mu \mathrm{m}$. C, D, Mean Gaussian-filtered spike trains (lower traces), corresponding raster plots (middle), and stimulus regimes (upper traces) for ipsilateral $(\boldsymbol{C})$ and contralateral eye stimulation $(\boldsymbol{D})$. Shaded and framed boxes and vertical lines as described in Figure 1. Scale bar, $100 \mu \mathrm{m}$. LAL, lateral accessory lobe.

CC neurons to those stimuli were either not present or not reproducible and were, therefore, not analyzed further.

\section{Looming sensitivity in neurons of the CBU}

Nineteen recordings from at least 10 different neuronal cell types with ramifications in the $\mathrm{CBU}$ showed looming sensitivity. These include a variety of tangential neurons, pontine neurons, and two types of columnar cells. Most neurons responded to ipsilateral and contralateral looming stimulation. Cell-specific responses were excitations or inhibitions to stimulation of either eye or spatially antagonistic responses, i.e., excitation to stimulation of one eye and inhibition to stimulation of the other eye (Table 1).

\section{Tangential neurons}

Looming sensitivity was found in seven types of tangential neuron of the CBU. Tangential neurons innervate particular layers of the central body. All neurons had smooth arborizations outside the central body; these ramifications were concentrated in the superior medial and intermediate protocerebrum and in the lateral accessory lobe. Axonal processes innervated particular layers of the $\mathrm{CBU}$ with beaded or varicose ramifications. Therefore, all of these tangential neurons can be regarded as input elements of the central body. Two recordings were from the giant fan-shaped neuron (GFS; Williams, 1975; Homberg, 1994). Two recordings were from neurons that were morphologically similar to the GFS neuron and were, therefore, termed GFS-like (GFSL1 and 2). One recording was from a cell type, termed TU2 by Homberg et al. (1999), and three recordings were from novel types of tangential neuron (TUx, TUy, TUz, Table 1).

All three novel neuron types (TUx, TUy, TUz) had input arborizations in the superior medial protocerebrum. The terminals 
of TUx (Fig. 2B) were concentrated in layer IIb of the CBU as defined by Heinze and Homberg (2008). Its primary neurite could be traced into the cell cortex adjacent to the inferior medial protocerebrum between the antennal lobe and the medial lobe of the mushroom body, but the soma was lost during tissue processing. The neuron showed excitatory responses to the looming stimulus presented to the ipsilateral and contralateral eye. In contrast, the neuron responded weakly or not at all to the display of the disc and during disc contraction (Fig. 2A,C,D). The neuron showed substantial adaptation in its response to repeated looming stimuli, a feature characteristic for many of the recorded neurons. The neuron was stimulated successively four times ipsilaterally and three times contralaterally with the same stimulus sequence containing a single looming event in each instance. After the neuron had adapted almost completely to the looming disc ipsilaterally we switched the monitors and repeatedly presented the stimulus sequence to the opposite eye. The neuron was again highly responsive to the looming stimulus but again adapted as during ipsilateral stimulation. This indicates that the adaptation process takes place already at an early stage upstream of TUx or at the dendritic level.

The main neurite of the TUy neuron projected in close proximity around the pedunculus (Fig. 3B). The neuron provided input to layer I of the CBU. Although TUy was also excited by stimulation of both eyes, the response was much stronger on the ipsilateral side (compare Fig. 3C,D). The third tangential neuron with ramifications in the superior medial protocerebrum, termed TUz, was inhibited by expansion of discs and squares on the ipsilateral side and excited when stimulating the contralateral eye (Fig. 4). The cell body of TUz was in the superior protocerebrum, just below the calyx of the mushroom body. Fine arborizations were confined to a restricted area in the superior medial protocerebrum. The axonal fiber projected via the anterior bundle to the central body and gave rise to beaded terminals in layers II and III of the CBU.

The GFS as well as the TU2 and the GFSL neurons had putative dendritic arborizations in the ipsilateral accessory lobe. The looming responses of the GFS neurons and those of the TU2 neuron were hardly noticeable on a single trial basis, because of their high background variability. In contrast, the GFSL neurons responded more strongly, and usually the looming response was clearly discernible from background on a single trial basis. Both GFSL neurons, one of the GFS neurons and the TU2 neuron, were binocular (Table 1). The GFSL neurons

B
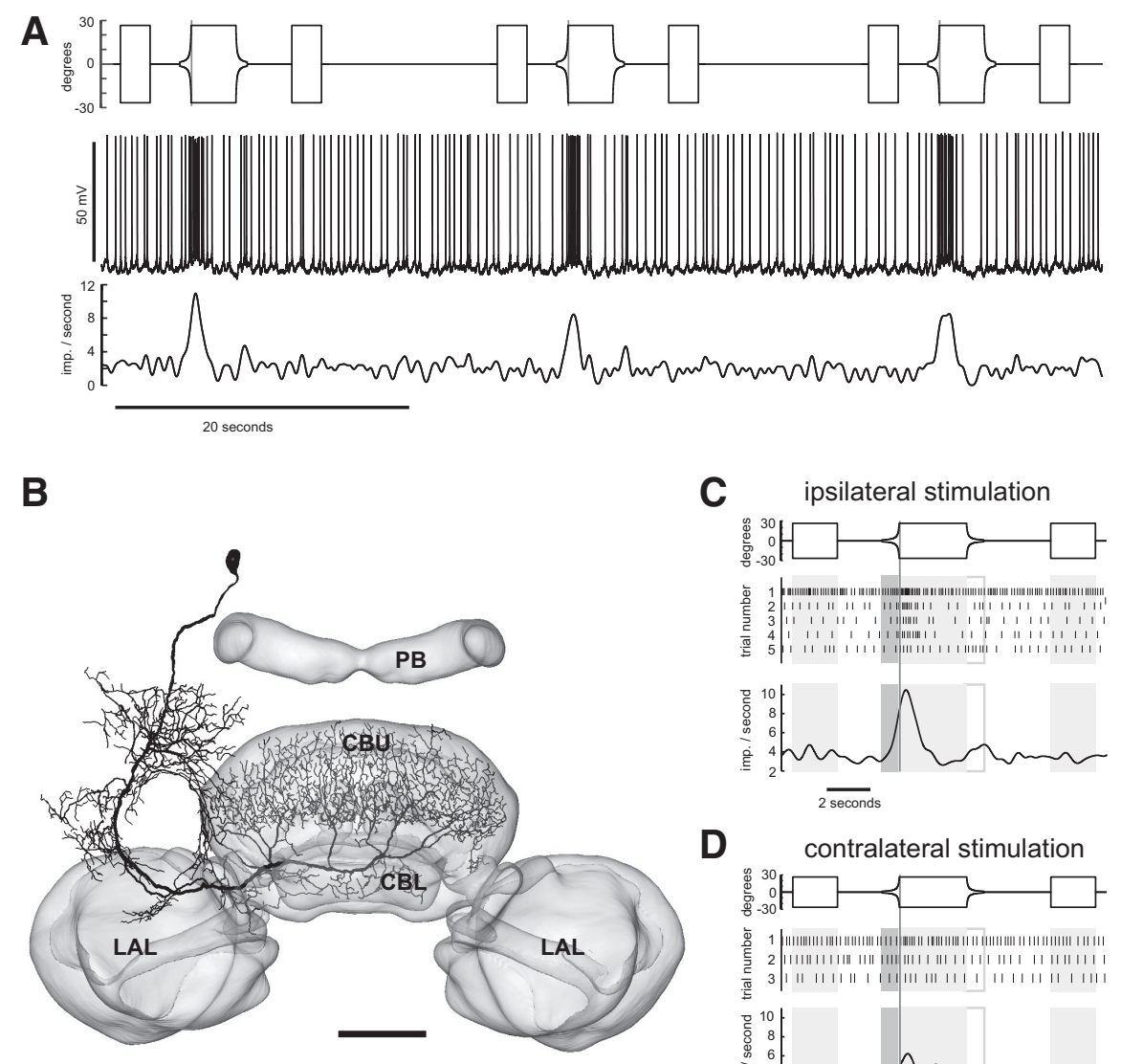

C ipsilateral stimulation

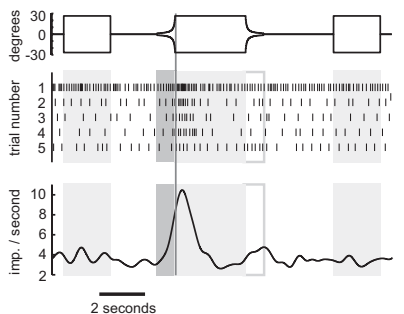

D

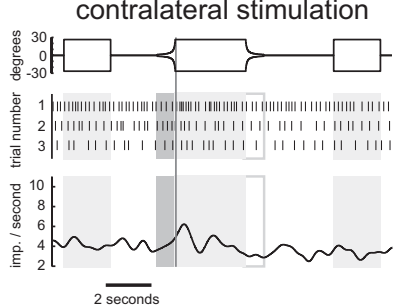

Figure 3. Looming-sensitive TUy tangential neuron of the CBU.A, Recording trace (middle) during stimulation of the ipsilateral eye. Lower trace shows Gaussian-filtered spike train. Upper trace shows angular extent of disc as a function of time. $\boldsymbol{B}$, Frontal reconstruction of the TUy neuron, registered into the standard central complex of the locust brain (el Jundi et al., 2010). C, D, Mean Gaussian-filtered spike trains (lower traces), corresponding raster plots (middle), and stimulus regimes (upper traces) for ipsilateral $(\boldsymbol{C})$ and contralateral eye stimulation (D). Shaded and framed boxes and vertical lines as in Figure 1. The three trials shown in A correspond to trials 2, 3, and 4 shown in C. LAL, lateral accessory lobe. Scale bar, $100 \mu \mathrm{m}$.
A

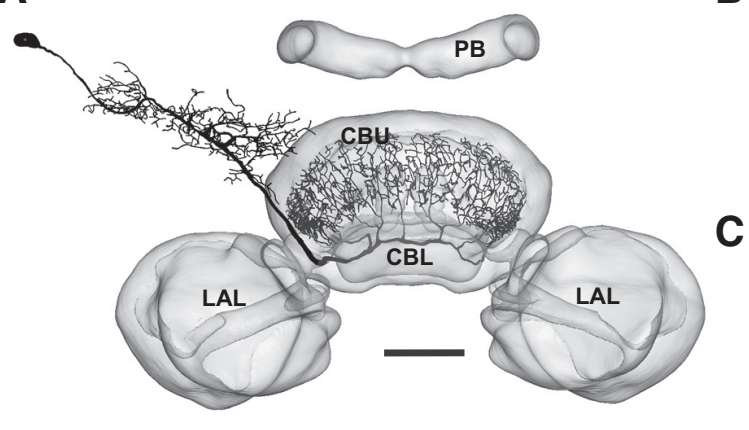

Figure 4. Spatially opponent responses to looming stimuli in the TUz neuron of the CBU.A, Frontal reconstruction of the neuron registered into thestandard central complex of the locust brain (el Jundi et al., 2010). B, C, Raster plots (top) and mean response traces (lower traces) during 4 resp. 5 presentations of stationary (shaded light gray) expanding (dark gray) and contracting (framed in gray) discs. The stimulus was applied to the ipsilateral ( $\boldsymbol{B}$ ) or contralateral ( $\boldsymbol{C}$ eye. Shaded and framed boxes represent time periods with presentation of stationary, expanding, and contracting disc as outlined in Figure 1. The cell responded to the looming stimulus on the ipsilateral side with reduced spiking frequency, but was excited when the stimulus was presented contralaterally. LAL, lateral accessory lobe. Scale bar, $100 \mu \mathrm{m}$.

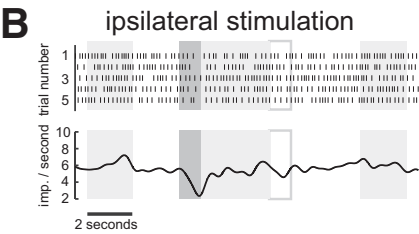

C

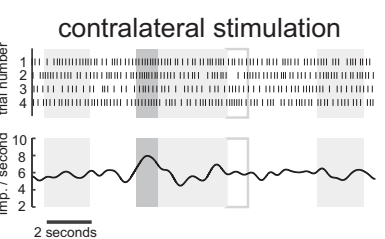

and the TU2 neuron were excited by the looming stimulus on either eye. In contrast, the GFS neurons were inhibited ipsilaterally and in one case additionally excited contralaterally (Table 1). 


\section{A}

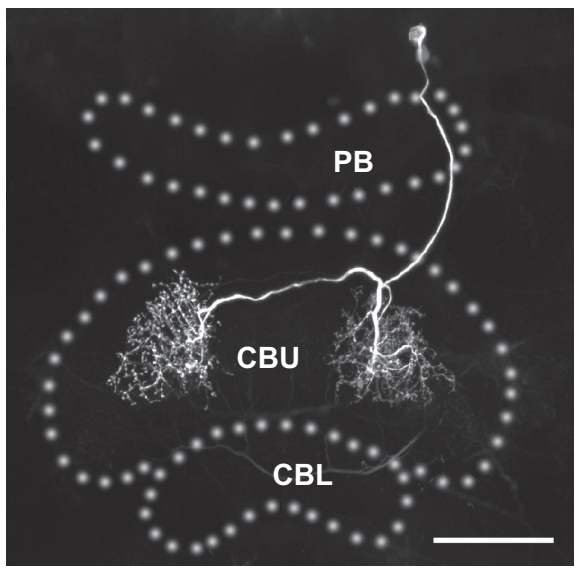

C

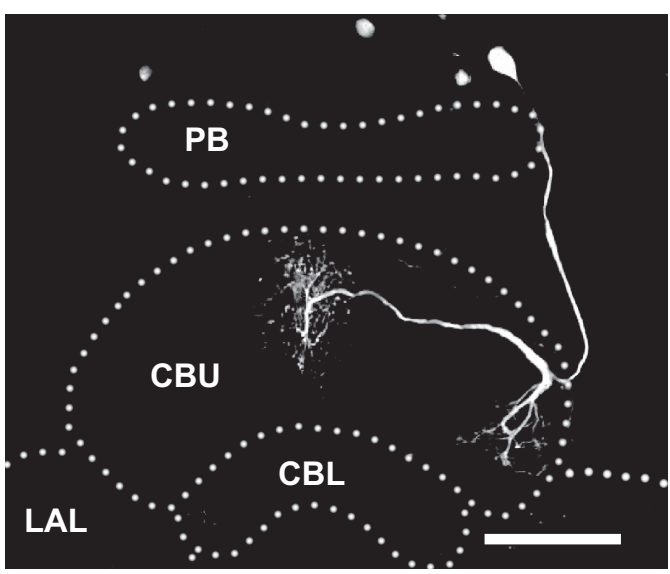

B

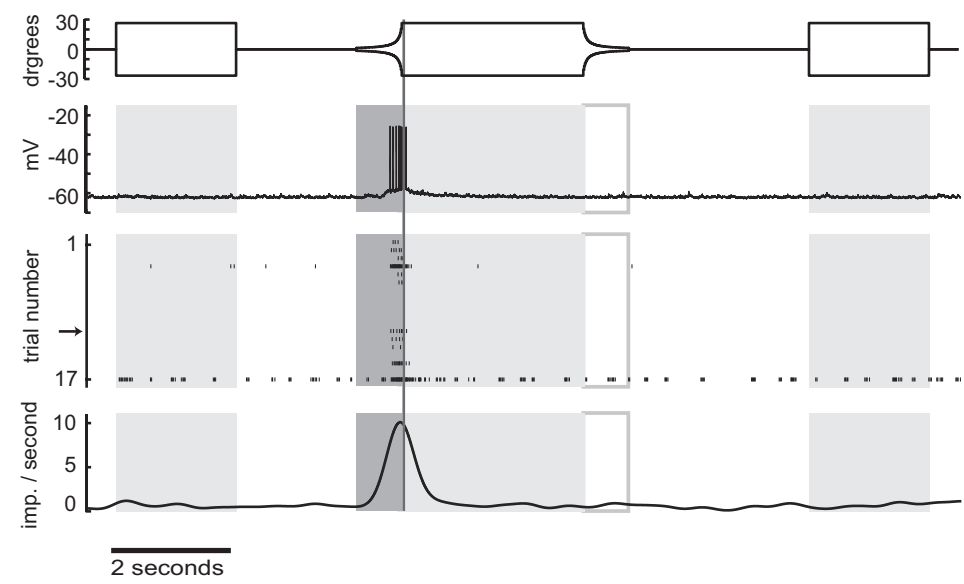

ipsilateral stimulation

D

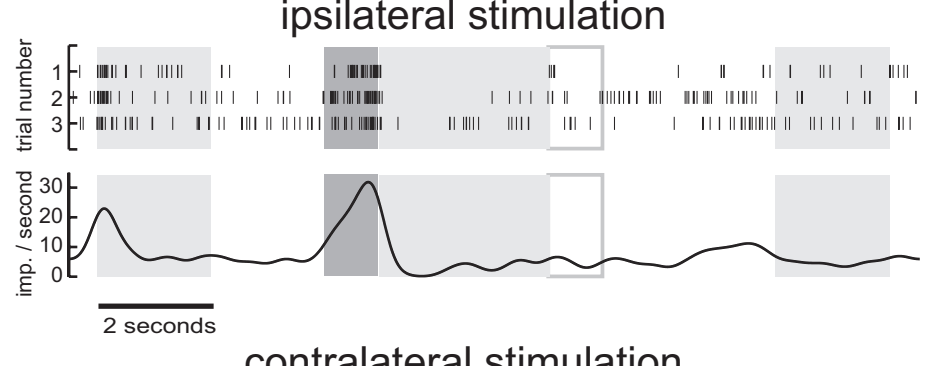

E

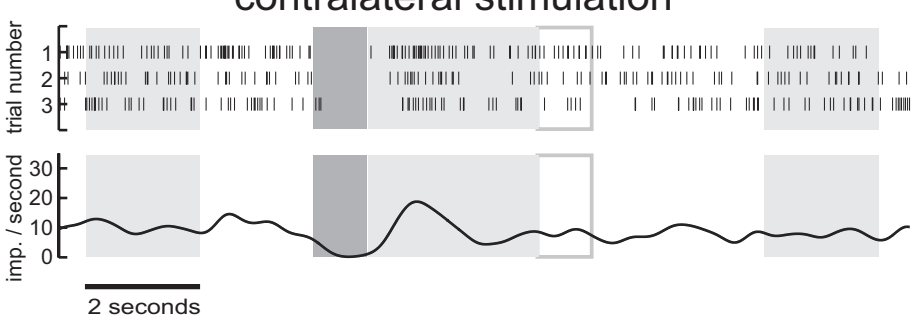

Figure 5. Azimuth-dependent responses of pontine neurons of the CBU. A, C, Maximum intensity projection (frontal view) of two PoU1 neurons ramifying in different columns of the CBU. B, Stimulus sequence and responses of the PoU1 neuron shown in $A$. Angular extent of disc as seen from the locustas a function of time (upper trace) and looming response (remaining traces) when stimulating the ipsilateral eye. Lower trace shows Gauss-filtered mean response of 17 trials, shown as raster plots above. In several trials spikes occurred only in response to the expanding disc. The trials without spikes followed a period of depolarizing currentinjection and were presumably the consequence of desensitization. Arrow indicates the trial corresponding to the original recording trace above the raster plot. $D, E$, Responses of the PoU1 neuron shown in C that connected the outermost column 1 to the innermost contralateral column 8. Raster plots and mean response traces for ipsilateral $(\boldsymbol{D})$ and contralateral $(\boldsymbol{E})$ eye stimulation. The neuron was excited when stimulated ipsilaterally and was inhibited when stimulated contralaterally by the expanding disc. LAL, lateral accessory lobe. Scale bars, $100 \mu \mathrm{m}$.

\section{Pontine neurons}

In addition to horizontal layers, the CBU can be divided into arrays of 16 vertical columns (Heinze and Homberg, 2008). Pontine neurons are intrinsic to the CBU. They have cell bodies in the pars intercerebralis and connect ipsilateral and contralateral columns over a distance of eight columns (Heinze and Homberg, 2008). Based upon different layer-specific arborization domains, three types of pontine neuron, termed PoU1-3, were distinguished by Heinze and Homberg (2008). PoU1 neurons were studied in two recordings and both showed looming sensitivity. One PoU1 neuron responded in a clear spatially opponent manner. It had ipsilateral input ramifications in the outermost column 1 of the CBU and provided outputs centered to the innermost contralateral column of the CBU (Fig. 5C). This cell was excited when stimulating the ipsilateral eye and was inhibited by contralateral stimulation (Fig. 5D,E). The second PoU1 neuron connecting ipsilateral column 4 of the CBU to column 5 in the contralateral hemisphere (Fig. 5A) had very low background activity. The neuron was excited when the ipsilateral eye was stimulated by a dark looming disc but did not show a response when the opposite eye was stimulated. The neuron often generated spikes only in response to the ipsilateral looming stimulus and was completely silent otherwise (Fig. $5 B$ ). Notably, no spike was generated when the locust was stimulated with a bright stationary or expanding disc in front of a dark background, a stationary or translating dark square in front of a bright background, moving or stationary gratings, or a stationary or rotating bar. Thus, the response of this cell was highly specific for an ipsilaterally presented black expanding object in front of a bright background. Owing to the virtually absent background activity, a possible inhibition to contralateral looming, as observed in the first PoU1 neuron, would not have been detected.

\section{Columnar neurons}

Columnar neurons have somata in the pars intercerebralis, innervate single, or small groups of columns and send axonal projections to subfields in the anterior lip region or the lateral 
accessory lobe adjacent to the central body (Heinze and Homberg, 2008). We recorded from a novel type of so-called CU neurons that was highly looming sensitive. CU neurons innervate single or pairs of CBU columns and send axons to the anterior lip (CU1), or widely to the anterior dorsal shell of one lateral accessory lobe (CU2) or both lateral accessory lobes (CU3) (Heinze and Homberg, 2008). We recorded two times from a novel subtype of $\mathrm{CU}$ neuron, termed CU4. CU4 neurons innervate single columns of the CBU but, unlike CU1-3, send axonal projections to small areas in the most lateral aspects of the of the lateral accessory lobe, adjacent to the lateral triangle (Fig. 6A,C). A CU4 neuron with ipsilateral CBU arborizations in columns $6 / 7$ and an axon to the contralateral accessory lobe was inhibited when presenting the looming stimulus ipsilaterally and excited when stimulating the contralateral eye (Fig. 6A,B). Another CU4 neuron (Fig. $6 C$ ) was excited regardless of whether the ipsilateral or contralateral eye was stimulated (Fig. 6D,Table 1). That neuron, in contrast to the first one, had contralateral ramifications in the $\mathrm{CBU}$ (columns 5/6), and sent an axon to the contralateral accessory lobe.

\section{A}

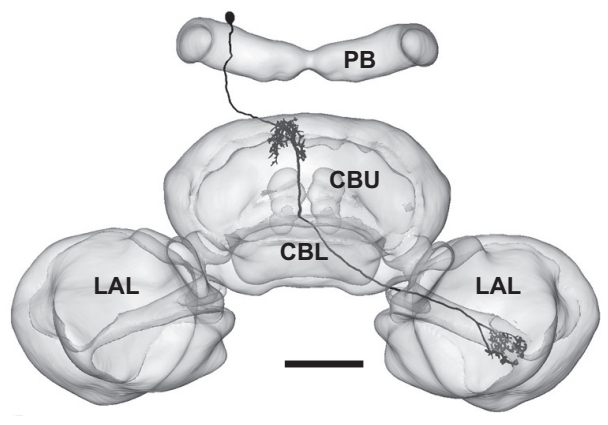

C

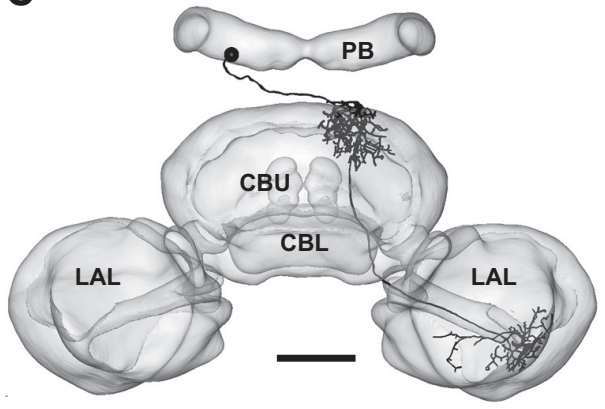

B ipsilateral stimulation

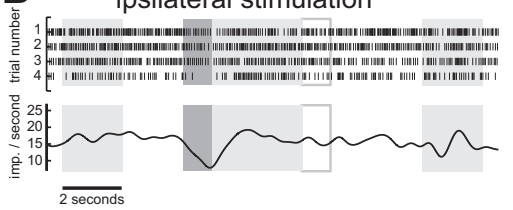

contralateral stimulation

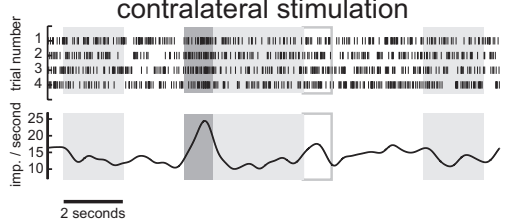

D ipsilateral stimulation

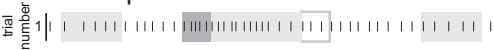

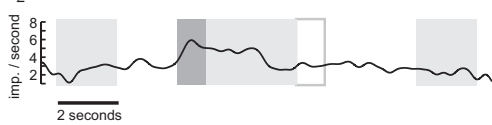

contralateral stimulation

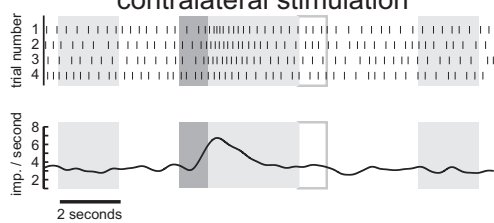

Figure 6. Azimuth-dependent responses of columnar neurons of the CBU. A, C, Frontal reconstruction of two CU4 neurons ramifying in different columns of the CBU. Neuronal reconstructions were registered into the standard central complex of the locust brain (el Jundi et al., 2010). Both neurons provide input to lateral aspects of the lateral accessory lobe. $\boldsymbol{B}, \boldsymbol{D}$, Responses of the CU4 neurons shown in $\boldsymbol{A}$ and $\boldsymbol{C}$. LAL, lateral accessory lobe. Scale bars, $100 \mu \mathrm{m}$.

\section{Looming sensitivity in neurons of the $\mathrm{CBL}$ and $\mathrm{PB}$}

We tested 19 neurons with ramifications in the CBL and/or in the $\mathrm{PB}$ that did not arborize in the CBU. All of these cell types have previously been shown to be sensitive to zenithal polarized light (Vitzthum et al., 2002; Heinze et al., 2009). All but one tested cell was looming sensitive (Table 1). One recording was obtained from a tangential neuron of the CBL. The neuron had its soma in the inferior medial protocerebrum and tangential ramifications in layer II of the CBL. Sparse ramifications in the median olive of the lateral accessory lobe are characteristic of TL3 tangential neurons. The neuron was inhibited when the locust was stimulated with expanding squares at the eye contralateral to the soma, but showed no looming response to ipsilateral stimulation.

We recorded 15 times from CL1 columnar neurons (Table 1). CL1 neurons innervate single columns in the PB and CBL and send axonal processes to the lateral triangle of the lateral accessory lobe. Most neurons showed excitatory responses to looming stimulation and were monocular or binocular. Most neurons responding to ipsilateral and contralateral stimulation showed different response amplitudes to stimulation of the right and left eye. Of the 15 CL1 neurons, three neurons were inhibited by the looming stimulus (Table 1). Their different response properties, however, were not reflected by obvious anatomical differences from the other neurons.

Three recordings were from tangential neurons of the $\mathrm{PB}$. One neuron could be identified as a TB1 tangential neuron, and two neurons belonged to the TB2 subtype. TB1 neurons have ramifications in the posterior optic tubercle, varicose terminals in one column in the ipsilateral and one column in the contralateral hemisphere of the bridge, and smooth rami- fications in 3-4 different columns in each hemisphere of the bridge. TB2 neurons are distinct from TB1 in that they have varicose processes in the innermost and outermost column of the bridge ipsilaterally and, in addition, a third domain of varicose arborizations in the innermost column of the contralateral hemisphere of the bridge (Heinze and Homberg, 2009). All TB neurons were excited by the looming stimulus. Two TB cells (one TB1 and one TB2) were excited regardless of whether the left or right eye was stimulated. The second TB2 neuron responded only when the ipsilateral eye experienced the looming stimulus.

\section{Looming sensitivity in premotor columnar output neurons of the $\mathrm{CC}$}

A particular class of columnar neurons, the CPU neurons (Heinze and Homberg, 2008), is thought to be the output elements of the CC that are presynaptic to descending motor pathways. CPU neurons integrate information provided by the $\mathrm{PB}$ and the CBU. Their putative output arborizations are in the ventral shell of one (CPU1 neurons) or both (CPU2 neurons) lateral accessory lobe(s). In the present account we recorded from four CPU1 neurons. CPU1 neurons have ramifications in single columns of the $\mathrm{PB}$, a pair of columns in layer I and II of the CBU, and send axonal fibers to large arborizations in the ventral shell of the contralateral accessory lobe. Heinze and Homberg (2007) and Heinze et al. (2009) showed that CPU1 neurons are sensitive to zenithal polarized light. We tested four CPU neurons for their sensitivity to expanding discs (Table 1, Fig. 7). Two of the tested cells were inhibited regardless of whether the looming stimulus was presented ipsilaterally or contralaterally (Fig. 7). One neuron was 
A

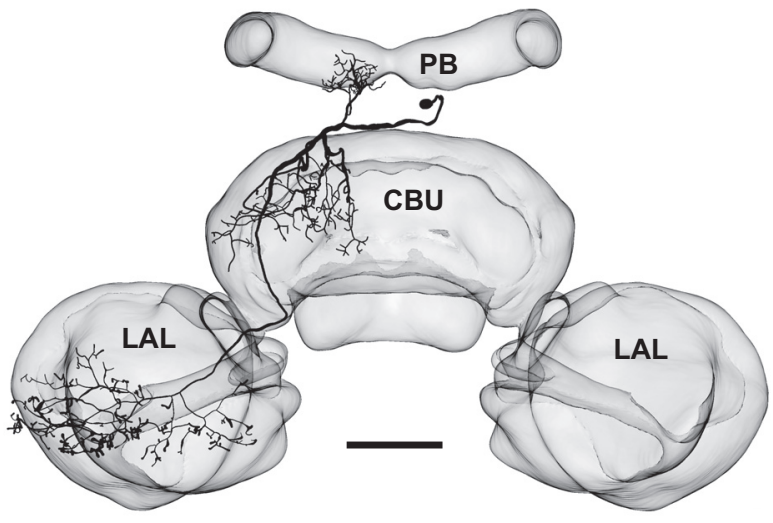

B
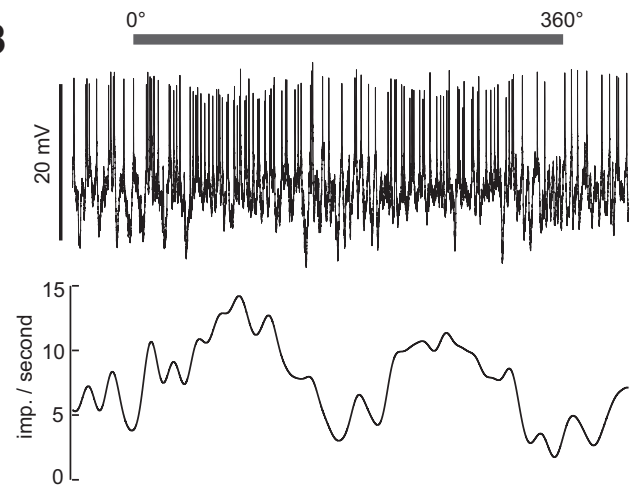

C
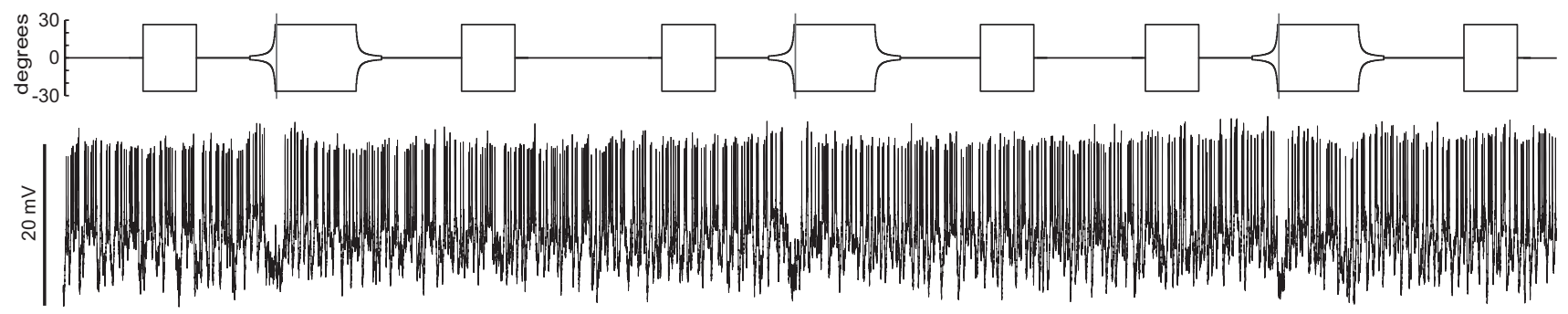

(10 seconds

D

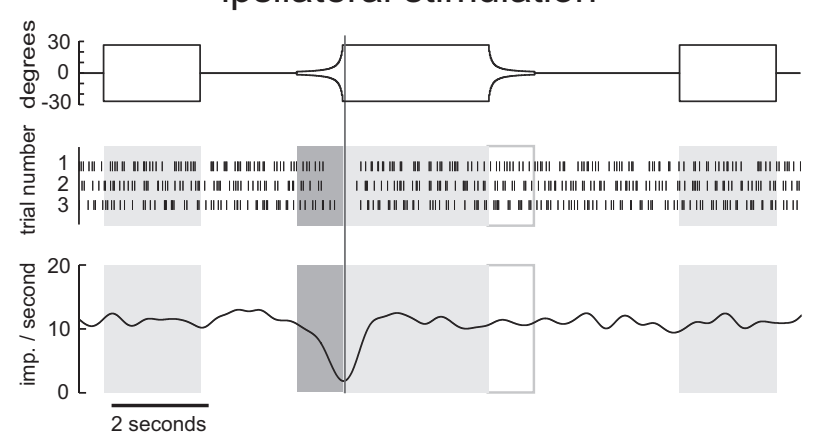

E

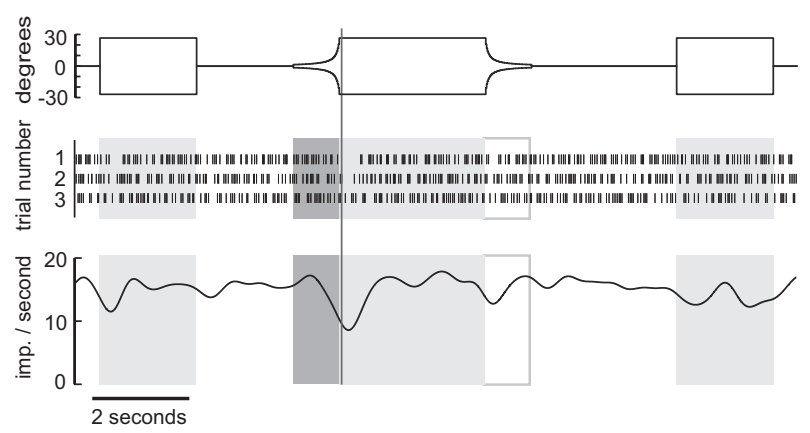

Figure 7. Output neuron of the $C C$ integrating information from the PB and the CBU. A, Frontal reconstruction of CPU1b neuron with smooth ramifications in the PB and the CBU and varicose endings in the ventral shell of the contralateral accessory lobe (LAL). Neuronal morphology registered into the standard central complex of the locust brain (el Jundi et al., 2010). Scale bar, $100 \mu \mathrm{m}$. $B$, Response of the neuron to dorsally rotated polarization filter, illustrated by gray horizontal bar. Top, Recording trace of the neuron during stimulation. Slow fluctuations removed by subtracting running average. Bottom, Gaussian-filtered spike train. C, Responses of the neuron to three repetitions of the looming stimulus. Top shows the angular extent of the disc as a function of time, middle shows the recording trace, and bottom shows the Gaussian-filtered spike train. $\boldsymbol{D}, \boldsymbol{E}$, Angular extent of the disc (top) raster plots (middle) and Gaussian-filtered spike trains (bottom) for responses to stimulation of the ipsilateral $(\boldsymbol{D})$ and contralateral eye $(\boldsymbol{E})$.

inhibited when experiencing the looming stimulus at the eye contralateral to the soma. In one case we only presented the looming stimulus ipsilaterally. Here the cell again was inhibited.

\section{Quantifying responses to looming stimuli}

For a first estimate of the timing of CC neuron reactions to the looming event we pooled the data from all recordings of looming sensitive neurons, including colabeled neurons (Table 1). We related the time of peak firing in those cells that were excited by the looming stimulus to the prospected time of collision of the simulated objects (Fig. 8). We estimated the peak firing rate by
Gauss filtering the responses with a filter kernel with $200 \mathrm{~ms}$ SD. The median responses preceded the time of collision by $8 \mathrm{~ms}$ (Fig. $8 A$ ). The DCMD response shown in Figure 1 peaked at $-170 \mathrm{~ms}$. In general, the times of peak firing in relation to time of collision differed considerably, even between different recordings from the same cell type (Fig. 8B). Response times even faster than that of the DCMD neuron were found in the two CU4 neurons $(-523$ $\mathrm{ms},-303 \mathrm{~ms})$. The two PoU1 neurons had response times of $-220 \mathrm{~ms}$ and $-87 \mathrm{~ms}$, and the three TB neurons, $-221,-58$, and $+64 \mathrm{~ms}$. In contrast, most CL1 neurons showed peak firing shortly after the time of collision, but two neurons were ex- 
tremely fast at $-454 \mathrm{~ms}$, respectively, $-522 \mathrm{~ms}$ (Fig. 8B). The morphological data did not allow us to determine whether these neurons were of a particular subtype of CL1 cells. Corresponding to the wide range of morphological types, TU neurons showed the largest variations in peak firing times ranging from -484 to +268 ms relative to time of collision.

\section{Responses of CC neurons to small translating objects}

We tested a subset of 18 CC neurons for their sensitivity to small stationary and moving squares. Sixteen (5) cells were tested with dark (white) squares against a bright (dark) background. Eleven neurons responded to moving squares, and seven neurons showed no response. Sensitivity to moving squares was found in tangential neurons of the $\mathrm{PB}$ and the $\mathrm{CBU}$, in a pontine neuron of the CBU and in columnar neurons with and without ramifications in the $\mathrm{PB}, \mathrm{CBU}$, and $\mathrm{CBL}$ (Table 1). Six neurons (three CL1 and three $\mathrm{TB}$ ) responding to moving squares had no ramifications in the CBU. All neurons responding to small object translations were also looming sensitive. Most CC neurons that were sensitive to moving squares responded most conspicuously to the first translation in a sequence but not to the stationary squares. Therefore, we classified a neuron as sensitive to small translating objects if it responded with the deepest drop or the highest peak spiking rate of the whole sequence during the first translation (Table 1).

Four responses from a TB1 neuron are shown in Figure 9A. We stimulated each eye with a dark rectangle in front of a bright background moving upward and downward and subsequently backward and forward. Pronounced responses as reflected in elevated spiking activity occurred only to the first upward translation and a much smaller response to the first backward motion (Fig. 9A, top). When applying the stimulus sequence in reversed order, starting with a downward translation instead of an upward motion, the highest spiking frequency was again observed when motion started (Fig. 9A, bottom). These data do not support directionality but suggest that the neuron serves as some kind of novelty detector with regard of the motion of small objects. Two further TB neurons were tested for their responsiveness to the small translating objects. Both cells were sensitive to the stimulus (Table 1). However, only one responded as strongly as the neuron shown in Figure 9A.

The response profile of a CU4 neuron differed strikingly from that of the remaining cells. This neuron was the only one showing reproducibly directionally sensitive responses to the translating squares (Fig. 9B). The neuron was excited by a moving square, but only when moving ventrally and when movement started in the center of the screen. In Table 1 this cell is marked as sensitive to small translating squares although it does not fulfill the strict criterion outlined above.

\section{Discussion}

A large variety of neurons of the locust CC are sensitive to looming stimuli and small translating targets. Neurons responsive to looming included 3 types of columnar neuron, 1 type of pontine neuron, and 10 types of tangential neuron, innervating all substructures of the CC except the noduli. Likewise, three types of columnar, one pontine, and four different tangential neurons responded to small moving squares. While cell types with ramifications in the PB and CBL (Table 1) were previously shown to be sensitive to the oscillation plane of polarized light suggesting a role in sky compass orientation (Heinze et al., 2009), the function of the CBU stayed elusive. We found a range of CBU neurons to be strongly looming sensitive. The results provide first indications for the potential general involvement of the CC in looming elicited behavioral responses in locusts.

\section{Visual integration in the CC}

The CC plays prominent roles in sensory motor integration, especially those related to spatial tasks. In locusts, crickets, and monarch butterflies skylight polarization signals are processed in the CC (Sakura et al., 2008; Heinze and Reppert, 2011; Homberg et al., 2011). In desert locusts, a compass-like representation of zenithal $E$-vector tunings in the PB suggests that the CC plays a role as an internal sky compass (Heinze and Homberg, 2007). Evidence for polarization-independent visual functions largely originates from behavioral experiments on mutant and transgenic flies (Drosophila melanogaster) with defects in parts of the CC. These studies suggest that CC neurons detect the orientation and elevation of edges (Liu et al., 2006), play a role in visually mediated spatial orientation memory (Neuser at al., 2008) and place memory (Ofstad et al., 2011), and are involved in the visual control of gap-crossing behavior (Triphan et al., 2010). Data from neuronal recordings substantiating these results are missing, however, except for a single study reporting responses to moving bars in the CC of flesh flies (Phillips-Portillo, 2012). We encountered only subtle or no responses to moving gratings or bars when testing these stimuli in preliminary experiments and sporadically in a subset of neurons presented here. 
A
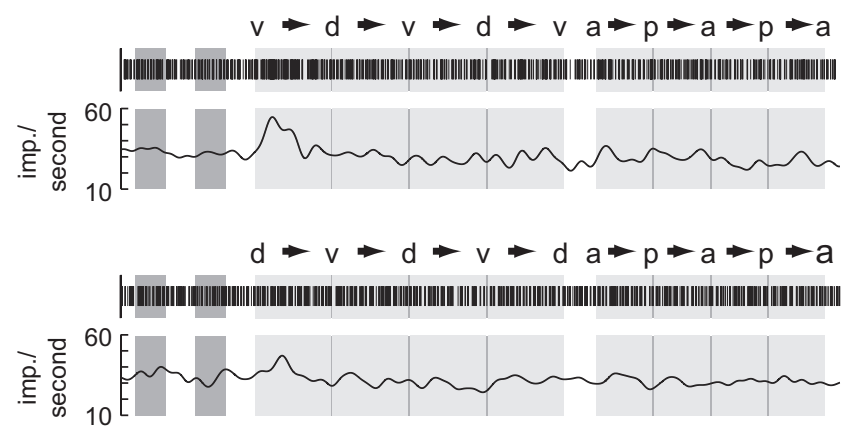

contralateral stimulation

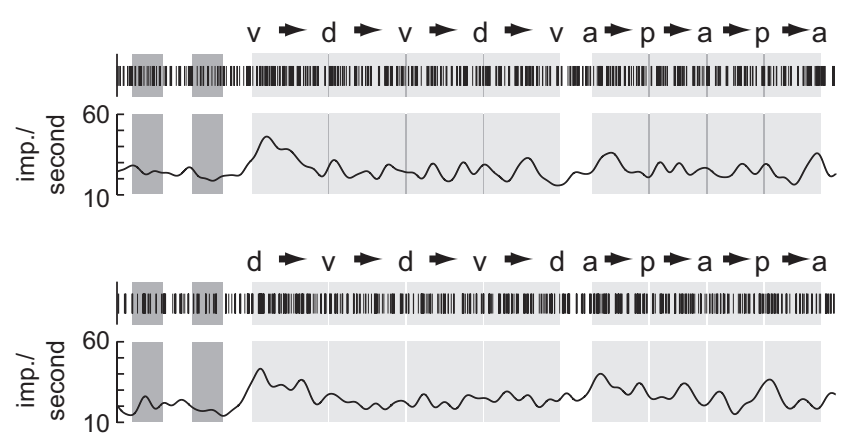

B $3 \overline{\text { seconds }}$

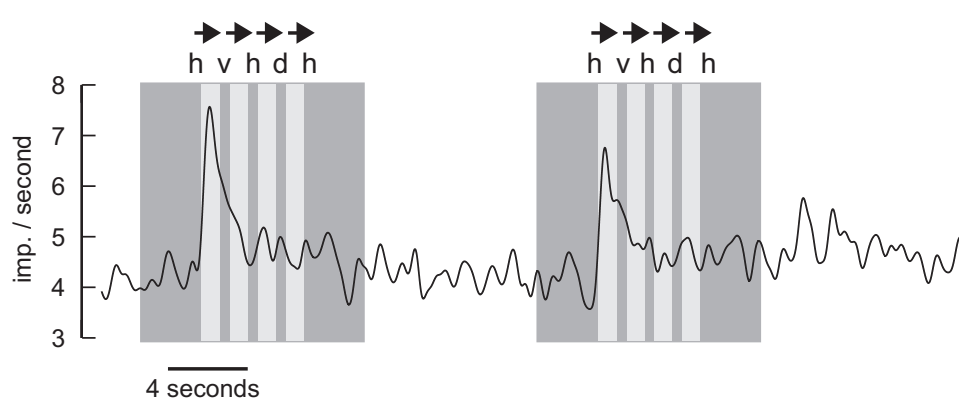

$3 \overline{\text { seconds }}$
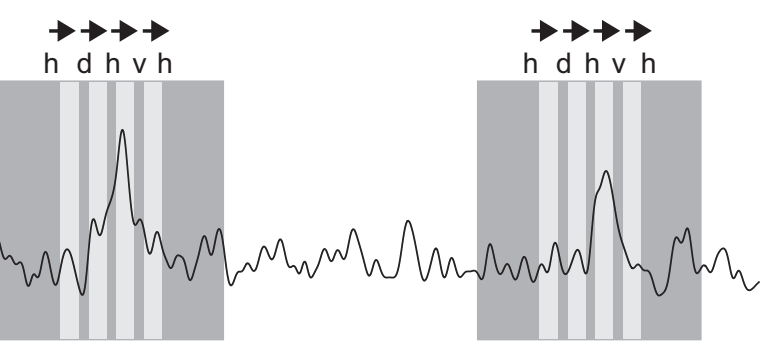

Figure 9. Sensitivity to small moving objects. $\boldsymbol{A}$, Raster plots and Gauss-filtered spike trains of the first-trial responses of a TB1 neuron to small black translating squares ( $8 \times 8^{\circ}$ in screen center) against a bright background. Dark gray (light gray; blank) areas represent time intervals when a stationary (moving; none) square was displayed. The first two dark areas represent time intervals during which the square was shown in the middle of the screen. This corresponds to an azimuth of $90^{\circ}$ and an elevation of $0^{\circ}$ when defining a position in front of the locust as being at an azimuth of $0^{\circ}$ and an elevation of $0^{\circ}$. Labels above the raster plots indicate the extreme positions of the square $\left(\mathrm{d}\right.$, dorsal, azimuth $0^{\circ}$, elevation $47^{\circ}$; v, ventral, azimuth $0^{\circ}$, elevation $-47^{\circ}$; a, anterior, azimuth $39^{\circ}$, elevation $0^{\circ} ; p$, posterior, azimuth $-39^{\circ}$, elevation $0^{\circ}$ ). Arrows indicate moving direction of the square. Note that in the top the square started moving ventrally while it started dorsally in the bottom. In each trial the neuron was excited in correlation with the stimulus only during the first vertical movements $(v \rightarrow d$; $d \rightarrow v$ ). In the contralateral trials there may also be an excitation during the first horizontal movement $(\mathrm{a} \rightarrow \mathrm{p})$. $\boldsymbol{B}$, Gaussian-filtered spike train of a CU4 neuron that responded in a directionally sensitive manner to a small, black translating square $\left(8 \times 8^{\circ}\right.$ in screen center) against a bright background. The eye contralateral to the cell body position was stimulated. Dark gray (light gray; blank) areas represent time intervals when a stationary (moving; none) square was presented. The square moved in a dorsoventral direction. $h, v, d$ represent horizontal $\left(0^{\circ}\right)$, ventral $\left(-39^{\circ}\right)$, and dorsal $\left(39^{\circ}\right)$ elevations of the square. Arrows indicate moving direction. The neuron was excited when the square moved ventrally starting from a horizontal position.

\section{Response specificity and variability}

The recorded neurons showed a wide range of looming responses extending from monocular/binocular excitations/inhibitions to opponent responses when stimulating the right and left eye. The response profile of a given neuron was usually robust, as shown by stimulus repetition, but some neurons habituated strongly and responded conspicuously only during the first stimulation. The amount of habituation in CC neurons might depend on the current internal state of the animal. Habituation of the LGMD/ DCMD pathway is, likewise, much stronger in resting than in tethered flying locusts (Rind et al., 2008). Neurons of the same type often responded in a similar way (e.g., CPU neurons; Table 1 ), but in certain cases differed in their responses (Fig. $6 B, D$ ). Whether striking differences in the responses of CL1 neurons (excitations vs inhibitions) correspond to different subtypes of these neurons could not be determined in the neuronal morphologies.

The times of peak firing rates relative to collision varied greatly even between different recordings from the same cell types (Fig. 8 ). It is generally assumed that tangential neurons (TU) constitute inputs, pontine $(\mathrm{PoU})$ and $\mathrm{TB}$ neurons intermediate, and columnar neurons (most CL1 subtypes, $\mathrm{CPU}, \mathrm{CU}$ ) outputs of the CC network (Heinze and Homberg, 2009). This scenario of a linear circuit, however, is not reflected in the response latencies of the neurons. Although some TU neurons responded faster than intermediate neurons (TB, PoU1) followed by even slower sig- naling output neurons (CL1), CU4 outputs and two CL1 neurons are among the fastest responding neurons with peak firing rates even preceding those of the DCMD. Different subsystems of neurons responsive to looming events may, therefore, serve completely different functions in the CC, with some neurons able to contribute substantially to behavioral responses to fastapproaching objects as used here, and others more likely involved in much slower behaviors.

\section{Functional significance of looming sensitivity in the CC}

A variety of biological functions may underlie the responses of CC neurons to expanding shapes and translating squares. In certain neurons, responses might represent corollary discharge signals that inform the CC about behavioral responses mediated by pathways bypassing the CC such as the LGMD/DCMD pathway (Simmons et al., 2010; Fotowat and Gabbiani, 2011). If the CC controls the general direction of locomotion by means of global environmental cues, corollary discharge signals might either shut off the associated motor commands generated in the CC or inform the CC about a particular course adjustment that needed to take place because of an immediate threat. Specifically, CPU neurons have been proposed as outputs of the CC contributing to flight steering with respect to polarization patterns of the sky (Heinze and Homberg, 2009). When an object approaches on a collision course, spiking in CPU neurons might be shut off and more direct neuronal pathways circumventing the central brain 
could overtake to elicit collision avoidance behavior. Interestingly, CPU1 neurons only showed inhibitory responses to looming or small field stimuli, favoring the "shut off" hypothesis, and responded to both polarized light and looming stimuli (Fig. 7). Pontine neurons (PoU1) responded particularly vigorously to looming stimulation (Fig. 5). Kunst et al. (2011) showed that activation of PoU1 neurons in grasshoppers inhibits the generation of calling songs. This further supports the shut-off hypothesis.

Alternatively, CC neurons might actively control behavioral responses to looming. Peak firing frequencies of many cells that were excited by the looming stimuli preceded the time of collision, and likewise, many neurons that were inhibited by the expanding shapes started to reduce their firing rate before the time of collision. These neurons might mediate collision avoidance behaviors or approaches toward objects, e.g., during landing, visually targeted climbing, or gap crossing. In fruit flies the CC plays an important role in gap crossing (Triphan et al., 2010). Although it was suggested that edge detection by CC neurons is important to mediate this behavior, the evaluation of looming information for distance estimation could also play a role. The same holds for visually mediated climbing movements. Niven et al. (2010) found that locusts walking on a horizontal ladder use visual information to control limb displacements. Looming information signaling depth and distance could ease the detection of the rungs during walking. In Drosophila different neuronal pathways have been suggested to mediate landing versus collision avoidance (Tammero and Dickinson, 2002). Collision avoidance is triggered when the animals experience image expansion from lateral directions while leg movements preparing for landing are elicited if the expansion occurs in the frontal visual field. The neurons we tested responded to lateral stimulation favoring a role in collision avoidance. Future experiments aimed at testing looming stimuli from the front may reveal whether an involvement of the CC in landing responses is also conceivable.

Avoidance responses are necessary to escape approaching predators and to avoid collisions with conspecifics in a swarm or with stationary obstacles encountered during locomotion. Escape responses in nonflying locusts (Santer et al., 2005) and flies (Card and Dickinson, 2008) are modular. When the animals are confronted with a looming threat they jump in a direction away from the stimulus. Leg movements determining jump direction are highly independent from the actual jumping movements suggesting that more than a single neuronal pathway mediate this behavior (Santer et al., 2005; Card and Dickinson, 2008). In locusts the LGMD/DCMD pathway is involved in mediating escape jumps (Fotowat and Gabbiani, 2011) and gliding responses (Simmons et al., 2010) during flight in response to looming objects. Especially the timing of take-off in escape jumps can be predicted from the timing of the DCMD peak firing rate (Fotowat and Gabbiani, 2011). However, these and other looming-sensitive neurons (Fotowat et al., 2009; Gray et al., 2010) discovered so far could not be linked to the directional component of escape responses. The jump direction in locusts is controlled by preparatory movements of their prothoracic and mesothoracic legs. Steering is accomplished by flexing the prothoracic leg contralateral to the looming event and ipsilateral to its eventual jump trajectory and extending the contralateral foreleg (Santer et al., 2005). Likely neuronal substrates for mediating such preparatory movements are neurons that differentiate between objects approaching from either eye. Certain CC cell types showed rightleft opponency in their response to looming. These neurons were inhibited or excited depending on which eye was stimulated and thus might signal the gross azimuthal direction from where the looming event occurred. Via downstream pathways they could mediate the flexion or extension of a leg depending on whether the cell was inhibited or excited.

The looming-sensitive neurons might, finally, contribute to spatially controlled hiding responses. When sitting on a vertical rod resembling a blade of grass, locusts hide behind that rod when experiencing looming stimuli or small translating objects that were similar to the stimuli applied here (Hassenstein and Hustert, 1999). These responses are considerably slower $(>200 \mathrm{~ms})$ than optomotor steering responses in flight $(60 \mathrm{~ms}$; Robert and Rowell, 1992).

\section{References}

Borst A, Bahde S (1988) Spatio-temporal integration of motion: a simple strategy for safe landing in flies. Naturwissenschaften 75:265-267.

Brainard DH (1997) The psychophysics toolbox. Spat Vis 10:433-436. CrossRef Medline

Card G, Dickinson MH (2008) Visually mediated motor planning in the escape response of Drosophila. Curr Biol 18:1300-1307. CrossRef Medline

Clements AN, May TE (1974) Studies on locust neuromuscular physiology in relation to glutamic acid. J Exp Biol 60:673-705. Medline

Egelhaaf M (2006) The neuronal computation of visual motion information. In: Invertebrate vision (Warrant E, Nilsson DE, eds), pp 399-461. Cambridge, UK: Cambridge UP.

el Jundi B, Homberg U (2010) Evidence for the possible existence of a second polarization vision pathway in the locust brain. J Insect Physiol 56: 971-979. CrossRef Medline

el Jundi B, Heinze S, Lenschow C, Kurylas A, Rohlfing T, Homberg U (2009) The locust standard brain: a 3D standard of the central complex as a platform for neural network analysis. Front Syst Neurosci 3:21. Medline

Fotowat H, Gabbiani F (2011) Collision detection as a model for sensorymotor integration. Annu Rev Neurosci 34:1-19. CrossRef Medline

Fotowat H, Fayyazuddin A, Bellen HJ, Gabbiani F (2009) A novel neuronal pathway for visually guided escape in Drosophila melanogaster. J Neurophysiol 102:875-885. CrossRef Medline

Giurfa M, Menzel R (1997) Insect visual perception: complex abilities of simple nervous systems. Curr Opin Neurobiol 7:505-513. CrossRef Medline

Gray JR, Blincow E, Robertson RM (2010) A pair of motion-sensitive neurons in the locust encode approaches of a looming object. J Comp Physiol A Neuroethol Sens Neural Behav Physiol 196:927-938. CrossRef Medline

Hassenstein B, Hustert R (1999) Hiding responses of locusts to approaching objects. J Exp Biol 202:1701-1710. Medline

Heinze S, Homberg U (2007) Maplike representation of celestial E-vector orientations in the brain of an insect. Science 315:995-997. CrossRef Medline

Heinze S, Homberg U (2008) Neuroarchitecture of the central complex of the desert locust: intrinsic and columnar neurons. J Comp Neurol 511: 454-478. CrossRef Medline

Heinze S, Homberg U (2009) Linking the input to the output: new sets of neurons complement the polarization vision network in the locust central complex. J Neurosci 29:4911-4921. CrossRef Medline

Heinze S, Reppert SM (2011) Sun compass integration of skylight cues in migratory monarch butterflies. Neuron 69:345-358. CrossRef Medline

Heinze S, Gotthardt S, Homberg U (2009) Transformation of polarized light information in the central complex of the locust. J Neurosci 29: 11783-11793. CrossRef Medline

Homberg, U (1987) Structure and functions of the central complex in insects. In: Arthropod brain: its evolution, development, structure, and functions (Gupta AP, ed), pp 347-367. New York: Wiley.

Homberg U (1994) Flight-correlated activity changes in neurons of the lateral accessory lobes in the brain of the locust Schistocerca gregaria. J Comp Physiol A Neuroethol Sens Neural Behav Physiol 175:597-610. CrossRef

Homberg U (2008) Evolution of the central complex in the arthropod brain with respect to the visual system. Arthropod Struct Dev 37:347-362. CrossRef Medline

Homberg U, Vitzthum H, Müller M, Binkle U (1999) Immunocytochemistry of GABA in the central complex of the locust Schistocerca gregaria: 
identification of immunoreactive neurons and colocalization with neuropeptides. J Comp Neurol 409:495-507. CrossRef Medline

Homberg U, Heinze S, Pfeiffer K, Kinoshita M, el Jundi B (2011) Central neural coding of sky polarization in insects. Philos Trans R Soc Lond B Biol Sci 366:680-687. CrossRef Medline

Horváth G, Varjú D (2004) Polarization patterns in nature and polarized light in animal vision. New York: Springer.

Kirchner WH, Lengler J (1994) Bees perceive illusionary distance information from rotating spirals. Naturwissenschaften 81:42-43. CrossRef

Klagges BR, Heimbeck G, Godenschwege TA, Hofbauer A, Pflugfelder GO, Reifegerste R, Reisch D, Schaupp M, Buchner S, Buchner E (1996) Invertebrate synapsins: a single gene codes for several isoforms in Drosophila. J Neurosci 16:3154-3165. Medline

Kunst M, Pförtner R, Aschenbrenner K, Heinrich R (2011) Neurochemical architecture of the central complex related to its function in the control of grasshopper acoustic communication. PLoS One 6:e25613. CrossRef Medline

Liu G, Seiler H, Wen A, Zars T, Ito K, Wolf R, Heisenberg M, Liu L (2006) Distinct memory traces for two visual features in the Drosophila brain. Nature 439:551-556. CrossRef Medline

Müller M, Homberg U, Kühn A (1997) Neuroarchitecture of the lower division of the central body in the brain of the locust Schistocerca gregaria. Cell Tissue Res 288:159-176. CrossRef Medline

Neuser K, Triphan T, Mronz M, Poeck B, Strauss R (2008) Analysis of a spatial orientation memory in Drosophila. Nature 453:1244-1247. CrossRef Medline

Niven JE, Buckingham CJ, Lumley S, Cuttle MF, Laughlin SB (2010) Visual targeting of forelimbs in ladder-walking locusts. Curr Biol 20:86-91. CrossRef Medline

Ofstad TA, Zuker CS, Reiser MB (2011) Visual place learning in Drosophila melanogaster. Nature 474:204-207. CrossRef Medline

Pelli DG (1997) The VideoToolbox software for visual psychophysics: transforming numbers into movies. Spat Vis 10:437-442. CrossRef Medline

Phillips-Portillo J (2012) The central complex of the flesh fly, Neobellieria bullata: recordings and morphologies of protocerebral inputs and small field neurons. J Comp Neurol 520:3088-3104. CrossRef Medline

Rind FC, Santer RD, Wright GA (2008) Arousal facilitates collision avoidance mediated by a looming sensitive visual neuron in a flying locust. J Neurophysiol 100:670-680. CrossRef Medline
Ritzmann RE, Harley CM, Daltorio KA, Tietz BR, Pollack AJ, Bender JA, Guo P, Horomanski AL, Kathman ND, Nieuwoudt C, Brown AE, Quinn RD (2012) Deciding which way to go: how do insects alter movements to negotiate barriers? Front Neurosci 6:97. Medline

Robert D, Rowell CHF (1992) Locust flight steering. I. Head movements and the organization of correctional manoevres. J Comp Physiol A Neuroethol Sens Neural Behav Physiol 171:41-51. CrossRef

Sakura M, Lambrinos D, Labhart T (2008) Polarized skylight navigation in insects: model and electrophysiology of e-vector coding by neurons in the central complex. J Neurophysiol 99:667-682. CrossRef Medline

Santer RD, Yamawaki Y, Rind FC, Simmons PJ (2005) Motor activity and trajectory control during escape jumping in the locust Locusta migratoria. J Comp Physiol A Neuroethol Sens Neural Behav Physiol 191:965-975. CrossRef Medline

Schmitt S, Evers JF, Duch C, Scholz M, Obermayer K (2004) New methods for the computer-assisted 3-D reconstruction of neurons from confocal image stacks. Neuroimage 23:1283-1298. CrossRef Medline

Simmons PJ, Rind FC, Santer RD (2010) Escapes with and without preparation: the neuroethology of visual startle in locusts. J Insect Physiol 56: 876-883. CrossRef Medline

Srinivasan MV, Poteser M, Kral K (1999) Motion detection in insect orientation and navigation. Vision Res 39:2749-2766. CrossRef Medline

Tammero LF, Dickinson MH (2002) Collision-avoidance and landing responses are mediated by separate pathways in the fruit fly Drosophila melanogaster. J Exp Biol 205:2785-2798. Medline

Triphan T, Poeck B, Neuser K, Strauss R (2010) Visual targeting of motor actions in climbing Drosophila. Curr Biol 20:663-668. CrossRef Medline

Vitzthum H, Müller M, Homberg U (2002) Neurons of the central complex of the locust Schistocerca gregaria are sensitive to polarized light. J Neurosci 22:1114-1125. Medline

Wehner R (1981) Spatial vision in arthropods. In: Handbook of sensory physiology, Vol VIC (Autrum HJ, ed), pp 287-616. Berlin: Springer.

Wicklein M, Sejnowski TJ (2001) Perception of change in depth in the hummingbird hawkmoth Manduca sexta (Sphingidae, Lepidoptera): comparing a model and looming neurons. Neurocomputing 38: 1595-1602. CrossRef

Williams JLD (1975) Anatomical studies of the insect central nervous system: a ground-plan of the midbrain and an introduction to the central complex in the locust, Schistocerca gregaria (Orthoptera). J Zool 176:67-86. 